LAWRENCE LIVERMORE NATIONAL LABORATORY

Nonlinear Optical Absorption in Laser Modified Regions of Fused Silica Substrates

\author{
Ardie D. Walser, Stavros G. Demos, \\ Michael Etienne, Roger Dorsinville
}

April 23, 2004

Optics Communications 


\title{
Nonlinear optical absorption in laser modified regions of fused silica substrates
}

\author{
Ardie D. Walser ${ }^{a}$, Stavros G. Demos ${ }^{b}$, Michael Etienne ${ }^{a}$, and Roger Dorsinville ${ }^{a}$ \\ ${ }^{a}$ Department of Electrical Engineering, The City College and Graduate \\ Center of CUNY, 140th St. and Convent Ave. N.Y.C. 10031 \\ ${ }^{b}$ Lawrence Livermore National Lab., 7000 East Ave., L-411, Livermore, CA 94551
}

\begin{abstract}
The presence of strong nonlinear absorption has been observed in laser modified fused silica. Intensity-dependent transmission measurements using 355-nm, 532-nm and 1,064-nm laser pulses were performed in pristine polished regions in fused silica substrates and in locations that were exposed to dielectric breakdown. The experimental results suggest that multi-photon absorption is considerably stronger in the modified regions compared to pristine sites and is strongly dependent on the excitation wavelength.
\end{abstract}


There are numerous applications of high purity silica $\left(\mathrm{SiO}_{2}\right)$ glass in modern optoelectronics. Its use ranges from being the key material for low-loss optical fibers to its utilization in optical windows in high vacuum confinement chambers. It has also been suggested that laser induced structural changes in glasses could be used to achieve 3-dimensional data storage [1]. With this broadening in the range of utilization, the specifications of this material have become more demanding, which keeps this material in the forefront of scientific research.

Fused silica is commonly used for the manufacturing of optical components and it has been the material of choice for large aperture optical elements that require UV transmission. In large aperture laser system, fused silica optics are required to withstand increasingly higher power densities without sustaining irrevocable damage leading to significant optical losses although small amounts of damage may not hinder performance [2]. Damage initiation under high power pulsed laser irradiation is believed to be the results of excessive absorption by various types of defects embedded in the materials during both manufacturing and exposure to laser radiation, and result in discrete damage sites, or pinpoints, on the surface or the bulk of materials at fluences much below the dielectric breakdown threshold of the pure material $[3,4]$. The presence of a large damage pinpoint density can give rise to beam losses and intensity modulations, ultimately impeding laser system performance [5]. One key issue in fused silica optics related to its performance in large aperture laser systems is associated with the growth in size of laser induced damage sites under subsequent near UV (355-nm) laser irradiation. This effect, known as "damage growth", can lead to failure of the material within a short period of time [6].

It has recently been shown that laser induced damage in optical materials with nanosecond pulses is associated with the formation of dense plasma accompanied by temperatures on the order of $1 \mathrm{eV}$ [7]. These extreme conditions lead to irreversible material modifications including mechanical failure and defect formations. In fused silica, spectroscopic studies of defects located at damage sites under femtosecond and nanosecond pulsed irradiation indicate a similarity in their optical properties suggestive that the dominant defect species are the same, independent of the pulse-width or laser wavelength $[8,9]$. It has 
been postulated that these defects are responsible for re-ignition of the damage process at pre-existing damage sites leading to damage growth [6].

Although damage initiation has been the focus of intense investigation since the early days of laser development [2,3], the early stage mechanisms under nanosecond laser irradiation leading to the formation of plasma have still not been resolved. Recent work suggests that defect-assisted multiphoton ionization may be a common mechanism for damage initiation [12]. It is the objective of this work to examine if defect assisted multiphoton absorption processes are present at damage sites in fused silica during high power laser irradiation. Such processes can be related to the plasma "re-ignition" and damage growth observed in fused silica. Identification of the process leading to damage growth is important in order to devise methods to mitigate this problem.

The experimental setup shown in the schematic diagram in Figure 1 was used to measure the intensity-dependent transmission in fused silica substrates. A single Gaussian beam obtained by a mode-locked Nd:YAG laser is tightly focused into the sample using a $10 \mathrm{~cm}$ focal length lens. This produces a $1 / \mathrm{e}^{2}$ beam radius $\omega_{0}$ of approximately $50-\mu \mathrm{m}$ at the focus. The laser spot size was obtained by measuring the laser energy as a function of the position of a metal blade, scanned across the laser beam. The laser produces 25-psec pulses at 1.06 $\mu \mathrm{m}$ with energies per pulse ranging from few $\mu \mathrm{J}$ to $30-\mathrm{mJ}$. Using second and third harmonic generation crystals, optical pulses at 532nm and $355 \mathrm{~nm}$ are generated with a $20 \mathrm{~Hz}$ repetition rate. The incident optical pulse energies were constantly monitored by reflecting about $8 \%$ of each pulse into the probe of an energy-meter before entering the fused silica substrate. The intensity of the incident beam is adjusted by an attenuation setup consisting of a half wave plate and two crossed polarized Glan-Thompson polarizers. Using a second energy meter, the light transmitted through the medium was measured as a function of the intensity of the incident beam.

The samples used in our experiments were $1-\mathrm{cm}$ thick, polished amorphous silicon dioxide substrates (Corning 7940, manufactured by flame hydrolysis). Damage spots were generated on and near the surface of the substrates using the third harmonic of a 3-ns Q-switched ND:YAG laser as described in reference [6]. The damage sites were initiated using a fluence of 
$24 \pm 4 \mathrm{~J} / \mathrm{cm}^{2}$. These sites were subsequently exposed to ten additional pulses at 10 $\mathrm{J} / \mathrm{cm}^{2}$ to increase their size through the re-ignition of the damage process (damage growth). The modified material, that is the focus of this investigation, was generated during the laser induced damage process in the crater of the damage site. The visible damaged areas were about 1-mm in diameter.

The experiments involved measurement of the intensity-dependant transmission in damaged and pristine regions of fused silica substrates. Figure 2 shows the experimental data for one of the fused silica samples where the normalized transmitted signal is plotted as a function of the incident pulse energy. The irradiance at all the wavelengths was varied in the range of 3-70 $\mathrm{GW} / \mathrm{cm}^{2}$. Due to the presence of scattering at damage sites and the nonuniformity of the scattering properties at (or within) each damage site, a different fraction of the incident light is transmitted through each point studied. To compensate for the variation in scattering, the data shown in all figures are normalized to the value of the transmitted signal for incident pulse energy of $550-\mu \mathrm{J}$. The measurements shown in figure 2 were taken at one spot in a damaged site and represent a typical example of our experimental observations.

The maximum energy per pulse used in this experiment was the upper limit for which the experimental data taken at lower energies could be consistently reproduced when the laser pulse energy was lowered after measurements at high power. More specifically, pulses with energy higher than $\approx 600-\mu \mathrm{J}$ at $355 \mathrm{~nm}$ resulted in alteration of the scattering signal when compared with prior measurements at the same or lower irradiation level. This suggested the initiation of structural changes and damage growth within the damage site as a result of exposure to pulse energies higher than $\approx 600-\mu \mathrm{J}$. Hence, the maximum pulse energy used in this experiment is just below the onset of damage growth under $355 \mathrm{~nm}$ irradiation using our laser system.

Figure 2 shows the intensity of 355-nm pulses transmitted through a damage spot as a function of the incident intensity demonstrating a nonlinear behavior (solid diamonds). On the other hand, using the same laser intensities but laser pulses at 532-nm (open triangles) and 1064-nm (solid squares), we found that the response is linear. When the same measurement is performed in 
pristine regions of the sample we found that the transmission was essentially linear at all three wavelengths used in our experiments $(532 \mathrm{~nm}, 1064 \mathrm{~nm}$ and $355 \mathrm{~nm}$ ). These results strongly suggest the presence of a nonlinear absorption process at $355 \mathrm{~nm}$, possibly two-photon absorption (TPA), in the modified region of the fused silica substrate.

Fig. 3 shows the transmission dependence on the incident intensity at 355$\mathrm{nm}$ for a pristine region (open circles) and a modified spot (solid squares). The solid line in figure 3 shows a fit to the transmission curve of $355-\mathrm{nm}$ pulses through a damage spot. The fit assumes an intensity dependent loss coefficient $\ln \left(I_{\text {tr }} / I_{\text {in }}\right)=-C-I_{\text {in }} \beta \delta$, where $C$ is the constant linear loss term, $\beta$ is the TPA coefficient, $\delta$ is the interaction length in $\mathrm{cm}$ and $\mathrm{I}_{\mathrm{tr}}$ and $\mathrm{I}_{\mathrm{in}}$ are the incident and transmitted intensities (in $\mathrm{W} / \mathrm{cm}^{2}$ ). A transmission measurement at very low intensity is used as the linear loss term $\mathrm{C}$. Good agreement with the experimental data is obtained for $\beta \delta=1.510^{-11} \mathrm{cmW}^{-1}$. This seems to confirm the TPA hypothesis. Based on the reported value of the TPA coefficient of fused silica at 355-nm $[14,15]$, the linear response observed in our experiments in the pristine polished region at all wavelengths is not surprising since the range of irradiance used ( $\mathrm{I}_{\mathrm{o}}$ $=0$ to $\left.70 \mathrm{GW} / \mathrm{cm}^{2}\right)$ is too low to alter the linearity of the response.

The experimental results clearly suggest that the TPA losses occur mainly at the damage spots. Earlier reports $[6,7,9,16]$ have suggested that the high temperature plasma created during the formation of the damage spot modifies the surrounding silica to produce a thin layer of defects associated with nonbridging oxygen hole center (NBOHC), oxygen deficiency center (ODC) and clusters of silicon. SEM examinations show that the damage sites consist primarily of a molten core, surrounded by a region of fractured material. X-ray tomography has allowed to identify a compaction layer that is approximately 10 microns thick with a density that is approximately $20 \%$ higher at the bottom of the damage crater [17].

The above experimental results suggest that the modified layer of fused silica is responsible for the observed TPA. Defect assisted ionization via multiphoton absorption can lead, in the presence of a high irradiance laser pulse, to cascade electron multiplication and the creation of dense plasma which results 
in additional absorption of laser light and energy deposition on the material. The relationship between the re-formation of plasma at damage sites under subsequent laser irradiation with damage growth has been documented elsewhere [6]. In our experiments we have observed that following the onset of the nonlinear behavior at about $400-\mu \mathrm{J}$ (at $355 \mathrm{~nm}$ ), damage growth is observed at about $600-\mu \mathrm{J}$ suggesting a direct relationship between these processes. The observation of nonlinear absorption followed by plasma formation should be present at shorter wavelengths $(532 \mathrm{~nm})$ but at higher pulse energies. The laser energies available using our laser system did not allow the observation of these anticipated effects.

It has been reported that annealing silica reduces the photoluminescence associated with optically produced damage sites $[8,18]$. This effect suggests the passivation of defects responsible for the observed photoluminescence. If the same defects are responsible for TPA, annealing should lead to reduction or removal of the nonlinear response for the laser intensities used in this experiment. We tested this concept by annealing damage sites using long exposures to laser irradiation. Figure 4 shows the transmission response of the fused silica substrates at a damaged spot for freshly modified spot and for an "old" modified spot which has been subjected to hours of repeated exposure to the $355-\mathrm{nm}, 20-\mathrm{Hz}, 600-\mu \mathrm{J}$ focused beam. For comparison a curve for a pristine region is also shown. As in the previous figures, the data shown are normalized to the value of the transmitted signal for the incident pulse energy of 550- $\mu \mathrm{J}$. The transmission dependence of the overexposed ("old") spot is clearly linear and indistinguishable from the polished pristine spot response. These results suggest that repeated exposure to the $25-\mathrm{ps}$, UV radiation at $355 \mathrm{~nm}$ has subjected the sample to a process equivalent to etching or/and thermal annealing, although a visual inspection did not reveal any obvious modifications to the damage spot. Based on these results we can make the assumption that most of the nonlinear absorption occurs within a very thin layer and that the interaction length is not longer than the depth of the compaction layer or about $\delta \sim 10 \mu \mathrm{m}$ [17]. This yields TPA values for the damage sites as high as $\beta \sim 1.510^{-6} \mathrm{cmW}^{-1}$. 
In conclusion, we have shown that damage sites in fused silica show nonlinear absorption properties at 355-nm arising from the presence of a layer of modified material. TPA effects may lead to additional absorption in existing small surface damage pits which in turn may lead to damage growth and optical component failure. This work does not allow for identification of which defect species known to be present at damage sites is responsible for TPA. Such information may be obtained by repeating these measurements using a tunable laser source and comparing the threshold for the observation of TPA to the absorption spectrum of the different defect species.

We thank Dr. Kennedy Reed and Dr. Sergei Kucheyev for stimulating discussions.

The authors gratefully acknowledge the support of the LLNL Research Collaborations Program for HBCUs \& MIs, the National Science Foundation, and the City College Faculty Award Program. This work was performed in part under the auspices of the U.S. Department of Energy by the University of California, Lawrence Livermore National Laboratory under contract No. W-7405Eng-48 through the Institute for Laser Science and Applications and the Materials Research Institute. 


\section{Figure Captions}

Figure 1. Experimental setup for the transmission measurements.

Figure 2. The transmission of high energy optical pulses at 355-nm, 532-nm and 1064-nm through the damaged region of a fused silica substrate as a function of the incident energy.

Figure 3. The transmission of high energy optical pulses at 355-nm through damaged region ( $\square)$ and a pristine site $(\mathbf{O})$ of a fused silica substrate as a function of the incident energy. The solid line shows a theoretical fit to the transmission curve at a damaged spot.

Figure 4. The transmission response of the fused silica substrates at a damaged spot for freshly modified "new" sample and for an "old" sample. For comparison a curve for a pristine region is also shown. 


\section{References}

1. Jianrong Qiu, K. Miura, K. Hirao, “Three dimensional optical memory using glasses as a recording medium through a multi-photon absorption process," Jpn. J. Appl. Phys., 37, 2263 (1998)

2. M. Runkel, A. K. Burnham, D. Milam, W. Sell, M. Feit, and A. Rubenchik, "The results of pulse-scaling experiments on rapid-growth DKDP triplers using the Optical Sciences Laser at 351 nm“ Proc. SPIE 4347, 359 (2001).

3. N. Bloembergen, "Laser induced electric breakdown in solids" IEEE J. Quantum Electron., 10, 375 (1974).

4. X. A. Shen, S. C. Jones, and P. Braunlich, "Intrinsic optical damage in $\mathrm{KBr}$ at 532 nm", Phys. Rev. Lett. 62, 2711 (1989).

5. A. K. Burnham, M. Runkel, M. D. Feit, A.M. Rubenchik, R. L. Floyd, T. A. Land, W. J. Siekhaus, and R. A. Hawley-Fedder, “Laser-Induced Damage in Deuterated Potassium Dihydrogen Phosphate" Applied Optics, 42, 5483, 2003.

6. Stavros G. Demos, Mike Staggs, Mark Kozlowski, “Investigation of processes leading to damage growth in optical materials for large-aperture lasers," Applied Optics 41, 3628 (2002)

7. C.W. Carr, H. B. Radousky, A. M. Rubenchik, M.D. Feit, S. G. Demos, "Localized dynamics during laser-induced damage in optical materials", Phys. Rev. Lett., In Press. (2004) 
8. S. Juodkazis, M. Wantanabe, H. Sun, S. Matsuo, J. Nishii, H. Misawa, M. Miwa, “ Optically induced defects in vitreous silica," Optics Letters 21, 1729 (1996)

9. S. O. Kucheyev, S. G. Demos, "Optical defect s produced in fused silica during laser-induced breakdown", Applied Physics Letters, 82, 3230, 2003

10. Glass, A. H. Guenther, "Laser induced damage of optical elements-a status report," Appl. Opt., 12, 661 (1973).

11. S. C. Jones, P. Braunlich, R. T. Casper, X. A. Shen, “Recent progress on laserinduced modifications and intrinsic bulk damage of wide-gap optical materials,"K. Ooka, T. Kishii, J. Non-Cryst. Solids, 3, 344 (1970).

12. C.W. Carr, H.B. Radousky, S.G. Demos, “Wavelength Dependence of Laser Induced Damage: Determining the Damage Initiation Mechanisms", Physical Review Letters, 91, 127402, 2003

13.L.W. Hrubesh, R.M. Brusasco, W. Grundler, M.A. Norton, E.E. Donohue, W.A. Molander, S.L. Thompson, S.R. Strodtbeck, P.K. Whitman, M.D. Shirk, P.J. Wegner, M.C. Nostrand, A.K. Burnham. "Methods for mitigating growth of laser-initiated surface damage on DKDP optics at $351 \mathrm{~nm}$ ", Proc. SPIE vol.4932, 180, 2003.

14. Borzio, W.Czaja, "Urbach tails in the absorption spectra of amorphous and crystalline $\mathrm{SiO}_{2}$ ", Philos. Mag. B63, 7 (1991)

15.R. DeSalvo, Ali A. Said, David J. Hagan, Eric W. Van Stryland, Mansoor Sheik-Bahae, "Infrared to ultraviolet measurements of two photon-absorption and $\mathrm{n}_{2}$ in wide band solid," IEEE J. of Quantum Electronics 32, 1324 (1996) 
16. Stavros G. Demos, Mike Staggs, “ Application of fluorescence microscopy for noninvasive detection of surface contamination and precursors to laserinduced damage." Applied Optics 41, 1977 (2002)

17. J. Wong, D. Haupt, J. H. Kinney, M. Stevens-Kalceff, A. Stesmans, J. Ferreira, E. Lindsey, I. Hutcheon, “ Morphology microstructure and defects in fused silica induced by high 3w (355nm) laser pulses," LLNL report UCRL-ID142167, January 2001; A summary can be found at Proceedings of SPIE 4347, $466(2001)$

18. M. Wantanabe, S. Juodkazis, H. Sun, S. Matsuo, H. Misawa, M. Miwa, R. Kaneko, "Transmission and photoluminescence images of three-dimensional memory in vitreous silica," Appl. Phys. Lett. 74, 3957 (1999) 


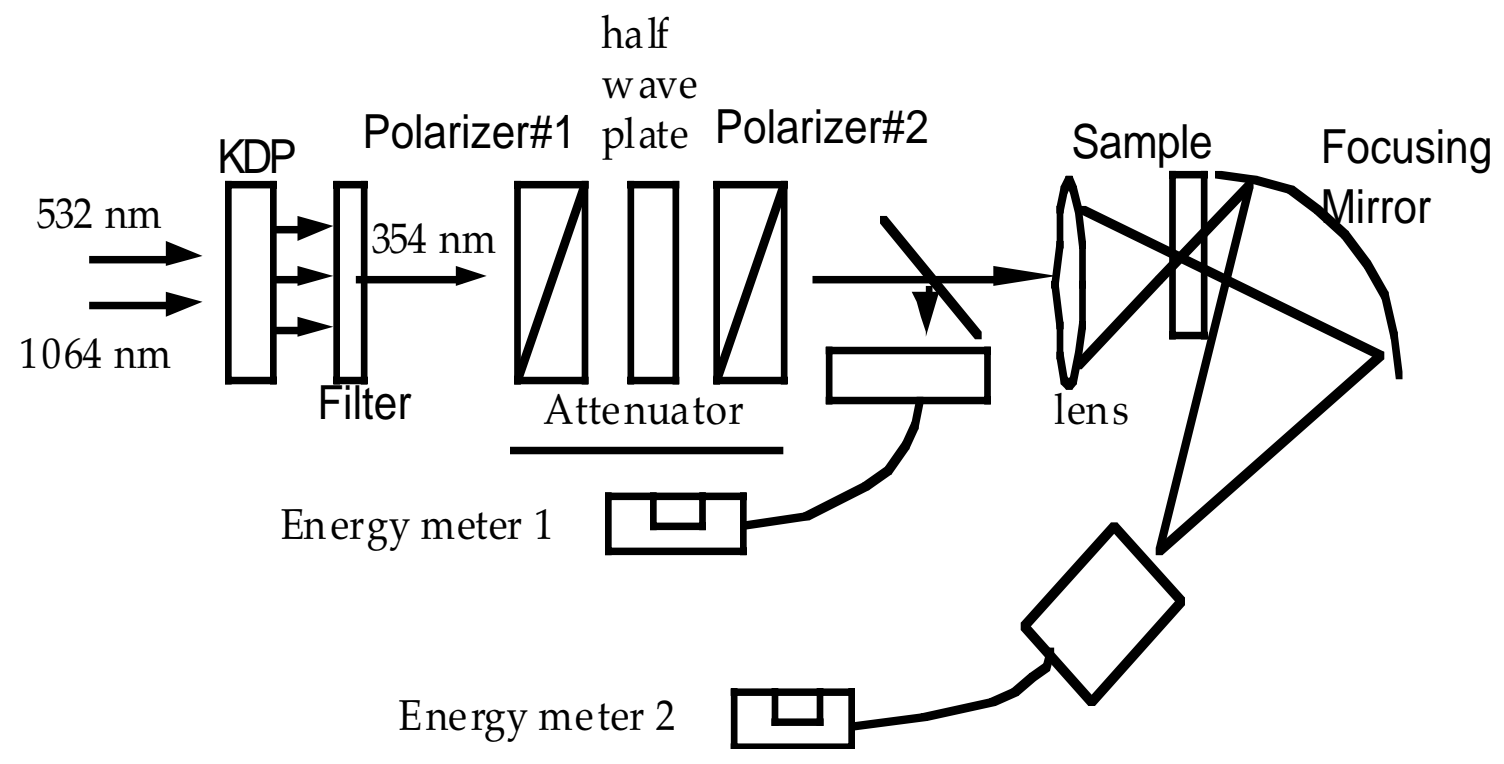

Figure 1 


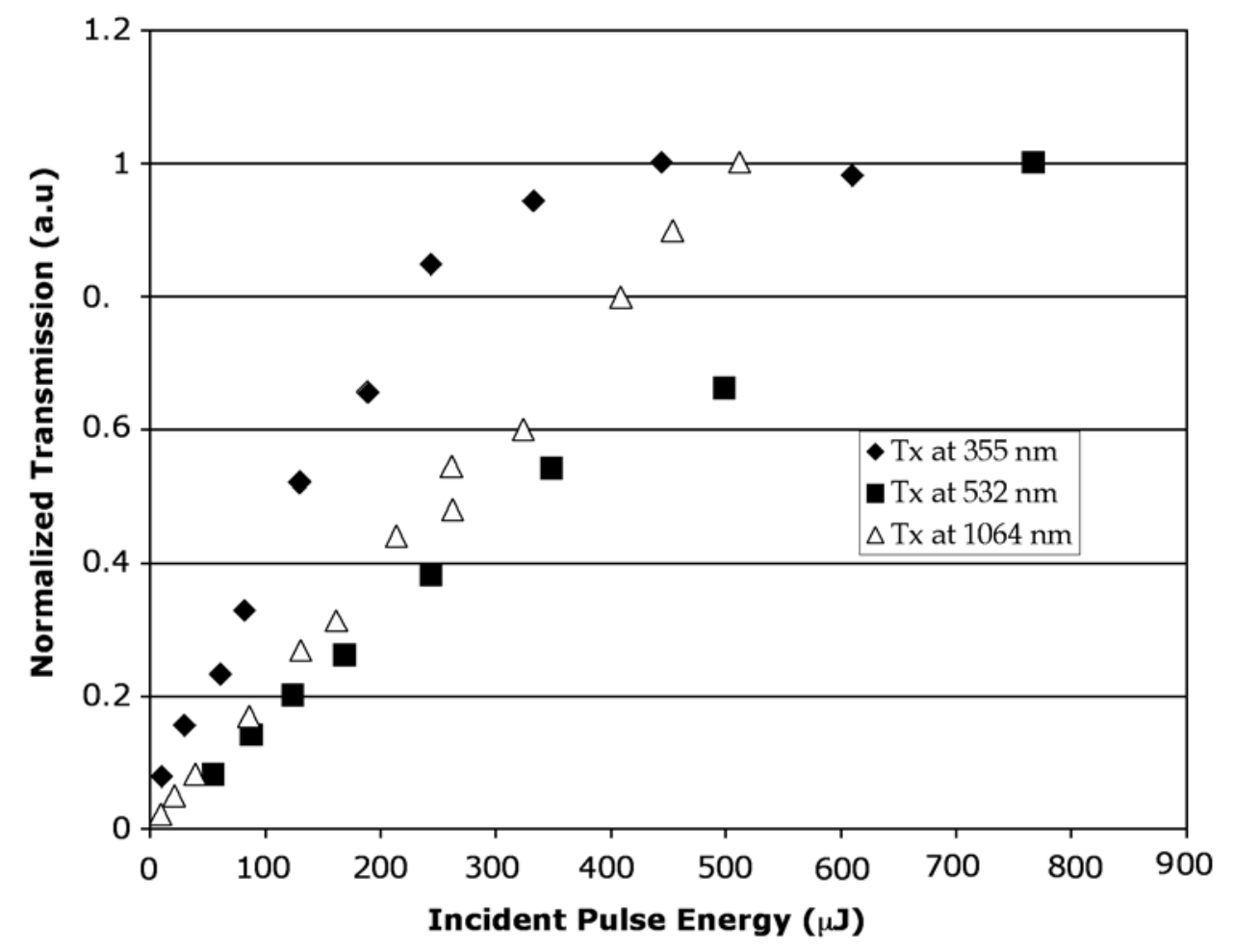

Figure 2 


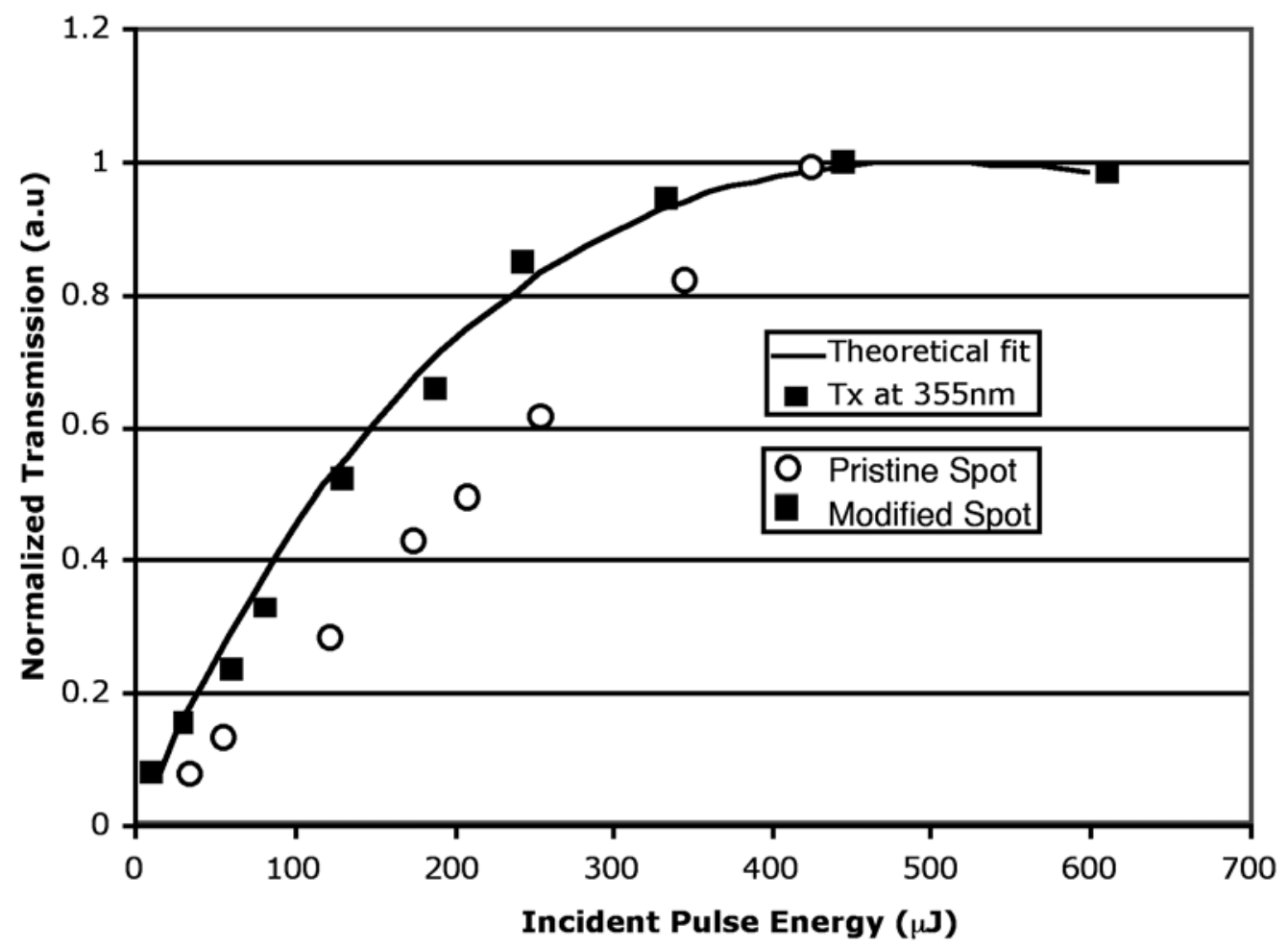

Figure 3 


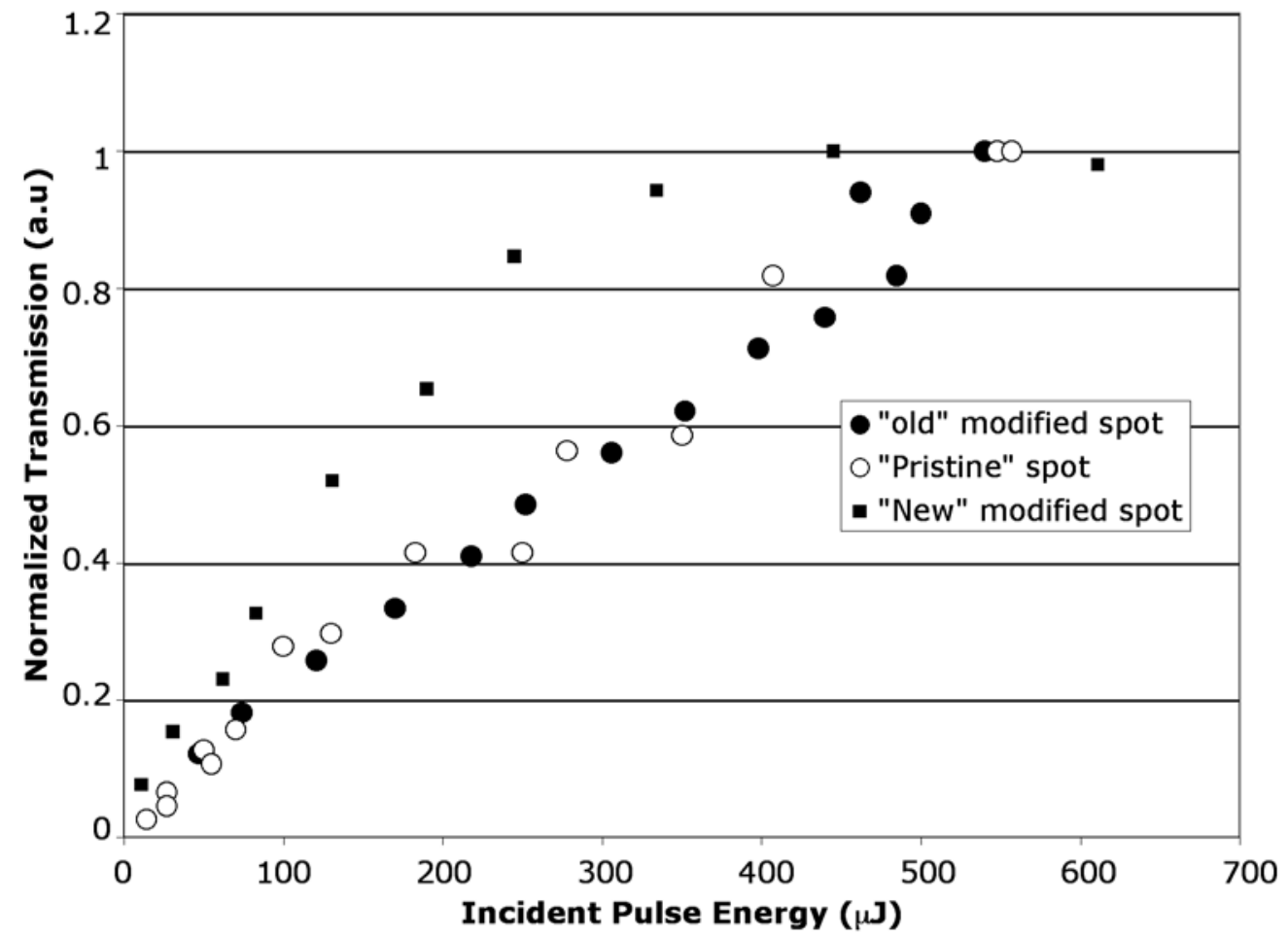

Figure 4 